\title{
GTRAP3-18 regulated by RNA-binding proteins under the control of microRNA
}

\section{Chisato Kinoshita, Toshio Nakaki}

\section{Department of Pharmacology, Teikyo University School of Medicine, Japan}

Glutathione (GSH) is a key antioxidant that plays an important neuroprotective role in the brain. Decreased GSH levels are associated with neurodegenerative diseases such as Parkinson's disease, Alzheimer disease and multiple system atrophy (MSA). We previously reported that rhythmic oscillation of miR-96-5p, one of the important microRNA, contributed the neuroprotection against oxidative stress through regulating the expression of excitatory amino acid carrier 1 (EAAC1) and GSH levels. Interestingly, there is a clinical report that have shown the expressions of miR-96-5p and its target EAAC1 are deregulated in the patient brains with MSA. These findings imply that dysregulation of miR-96-5p causes MSA through down-regulation of EAAC1 and reduction in GSH levels. In this study, we focused on GTRAP3-18, the negative factor of EAAC1, as a new target of miR-96-5p.

First, we investigated whether the expression of GTRAP3-18 is affected by manipulation of miR-96-5p level using western blot analysis and luciferase reporter gene assay. Since it turned out GTRAP3-18 to be indirectly regulated by miR -96-5p, we next identified the candidates of GTRAP3-18 regulators which can regulate the expression of GTRAP3-18 and be regulated by miR-96-5p using mass spectrometry analysis. Then, we have tested whether these candidate proteins could be a direct regulator of GTRAP3-18.

The result shows that GTRAP3-18 is up-regulated by miR-96-5p at transcriptional and translational levels. Since microRNAs negatively regulate gene expression in most cases, up-regulation of GTRAP3-18 by miR-96-5p is suggested to be mediated by some factors such as RNA binding proteins. We have identified several miR-96-5p-regulating RNA binding proteins that negatively regulate GTRAP3-18.

GTRAP3-18 could be regulated by RNA binding proteins that are modulated by miR-96-5p. 